\title{
Nanoscale
}

Check for updates

Cite this: Nanoscale, 2018, 10, 21842

\section{Onset of vertical bonds in new GaN multilayers: beyond van der Waals solids $\uparrow$}

\author{
A. Onen, ${ }^{a}$ D. Kecik, (D) b,a E. Durgun (D)*a and S. Ciraci*b
}

A suspended single layer of GaN in a honeycomb structure is stable in a planar geometry. By stacking these GaN layers one can construct bilayers or multilayers, even new three-dimensional (3D) periodic structures. In this study, we clarified how the planar layers are buckled with the onset of vertical Ga-N bonds. Among the four stable phases of bilayer GaN, only one of them maintains the planar geometry, which is bound by weak van der Waals interactions. For the remaining three phases, the layers are buckled with the onset of weak vertical bonds, and attain total energies slightly lower than that of the planar geometry. Structural phase changes, as well as direct-indirect band transitions take place under strain and electrostatic charging. The vertical bonds become shorter in multilayers, and eventually attain the bulk value. Among the stable phases of 3D periodic GaN, only one with a graphite-like structure behaves as a layered, van der Waals solid; whereby others are 3D uniform crystals beyond the van der Waals solid.

Received 12th July 2018,

Accepted 30th October 2018

DOI: $10.1039 / c 8 n r 05626 a$

rsc.li/nanoscale

\section{Introduction}

Gallium nitride (GaN) crystal in wurtzite structure (wz-GaN) is an important wide band-gap semiconductor with a critical and wide range of technological applications in microwave communications, lasers, detectors, and light-emitting diodes in the ultraviolet range. ${ }^{1-3}$ As early as 2005, first-principles calculations predicted that the GaN compound can form the freestanding, graphene-like, single-layer (SL) h-GaN structure. ${ }^{4}$ Later, extensive analyses of dynamical ${ }^{5}$ and thermal stability ${ }^{6}$ have demonstrated that free-standing, SL h-GaN is, in fact, stable in the planar honeycomb structure. Planarity, which is common to SL compounds like h-BN, h-GaN, and h-AlN that involve at least one element from the first row of the Periodic Table, is maintained by $\pi$-bonds between p-orbitals of the nearest cation and anion atoms. Conversely, silicene, germanene, and h-GaAs are buckled. ${ }^{5,7,8}$ Recently, numerous theoretical studies unveiled diverse aspects of $\mathrm{h}-\mathrm{GaN} ;{ }^{9}$ in particular they predicted that stable bilayer, multilayer and layered allotropes of h-GaN can form with tunable optoelectronic properties. ${ }^{6,9-12}$ Much recently, the synthesis of ultrathin twodimensional (2D) h-GaN on SiC via graphene encapsulation

\footnotetext{
${ }^{a}$ UNAM - National Nanotechnology Research Center and Institute of Materials Science and Nanotechnology, Bilkent University, Ankara, 06800, Turkey. E-mail:durgun@unam.bilkent.edu.tr

${ }^{b}$ Department of Physics, Bilkent University, 06800 Ankara, Turkey.

E-mail: ciraci@fen.bilkent.edu.tr

$\dagger$ Electronic supplementary information (ESI) available. See DOI: 10.1039/
} C8NR05626A method by Al Balushi et al. ${ }^{13}$ has brought $2 \mathrm{D}$ GaN further into focus.

While multilayers of 2D GaN, AlN, SiC, silicene, and germanene can be constructed, the important question of whether these materials are similar to van der Waals (vdW) solids with weak interlayer coupling leading to wide interlayer spacings, or if vertical chemical bonds may occur, gained significant importance. In fact, layered silicite ${ }^{7,8,14,15}$ and germanite structures with vertical covalent bonds were proposed to explain the data taken from multilayers of silicene and germanene. So far the structures of multilayer silicene and germanene have not been fully resolved. Similarly, SL h-GaAs is buckled; ${ }^{4,5}$ vertical bonds form readily in its multilayers. Incidentally, it was argued that the graphitic structure of few-layer planar h-GaN is unstable and reconstructs into a structure with specific vertical bonds, whereby alternating square and octagon rings appear, nevertheless the in-plane buckled honeycomb structure is maintained. ${ }^{16}$ Haeckelite structures in single and multilayer square-octagon GaN have been predicted to be stable. ${ }^{17-21}$ The analysis of electronic charges revealed formation of weak, covalent + ionic vertical bonds. ${ }^{21}$

Onset of the vertical bonds with a mixed covalent + ionic nature between specific cation and anion pairs in adjacent planes with $\mathrm{AA}^{\prime} \mathrm{A}$.. conformation, while expanding the distance between other pairs, is a complex interplay between $\mathrm{sp}^{2}-\mathrm{sp}^{3}$ hybridization dictated by the energy minimization. Their multilayers constructed by stacking of planar, SL graphene, h-BN and h-AlN remain planar with wide interlayer distances coupled with weak vdW interactions, forming typical vdW solids. Therefore, it is of current interest to know whether multilayers of planar, SL h-GaN can construct layered structures 
with weak interlayer coupling like vdW solids. Answering this question is very critical in the sense of paving the way towards new artificial three-dimensional (3D) layered crystals, or thin films exhibiting novel physical properties different from the parent 3D homogeneous crystals.

In this study, we examined the onset of vertical bonds in bilayer (B), trilayer (T), and periodic multilayers of h-GaN, and their effects on the electronic structure within first-principles density functional theory (DFT) calculations. Firstly, the nature of these vertical bonds in diverse multilayers of GaN is analyzed. We found that both planar bilayer (B-PL) and buckled, haeckelite-type bilayer (B-HK) phases possess dynamical and thermal stability, with a minute gain of energy by the onset of vertical bonds upon transforming from B-PL to B-HK, within the accuracy limits of DFT. However, the energy difference between them increases with increasing number of layers. The structural transition from B-PL to B-HK cannot occur spontaneously; it requires an external excitation to overcome the energy barrier between the two phases. Surprisingly, in contrast to what has been assumed so far, ${ }^{16}$ the haeckelite structure is not the lowest energy structure; instead there are manifolds of bilayer structures, which are energetically favorable. These phases of GaN bilayers display interesting structural changes and also direct-indirect band gap transitions by the applied strain and electrostatic charging. We also found that layered, as well as new 3D bulk structure can be constructed despite the fact that $3 \mathrm{D}$ wurtzite structure is a global minimum. Another striking, insofar surprising result is that all manifolds of bilayer and 3D periodic structures mark similar fundamental band gaps despite their significant structural differences set by the vertical bonds.

\section{Method}

The theoretical predictions of the ground state properties of $2 \mathrm{D}$ and periodic/bulk forms of GaN discussed in this paper are based on first-principles calculations performed using DFT. In the majority of the calculations presented, the plane-wave basis set together with the projector-augmented-wave potentials (PAW) formalism ${ }^{22,23}$ implemented in the Vienna $a b$ initio simulation package (VASP) ${ }^{24,25}$ is used. The electron exchange and correlation potential is described by the Perdew-BurkeErnzerhof (PBE) form within the generalized gradient approximation (GGA), with d-electrons also taken into account (GGA-d XC potential). ${ }^{26,27}$ Van der Waals interactions are accounted for the layered, periodic and bulk structures using the Grimme method. ${ }^{28,29}$ Atomic positions are optimized using the conjugate gradient (CG) method; the total energy and atomic forces are minimized with specific criteria for energy difference convergence. Monkhorst-Pack ${ }^{30} k$-point sampling adopted for the PBE calculations of the $2 \mathrm{D}$ unit cell is $25 \times$ $25 \times 1$, and $19 \times 19 \times 19$ for the periodic and bulk structures.

Various energy criteria for quantifying the interactions can be summarized as: the cohesive energies of layered allotropes of GaN relative to their constituent free atoms are calculated from the expression, $E_{\mathrm{c}}=E_{\mathrm{T}}[\mathrm{Ga}]+E_{\mathrm{T}}[\mathrm{N}]-E_{\mathrm{T}}[\mathrm{GaN}]$ in terms of the total energies $E_{\mathrm{T}}[\mathrm{Ga}]$ and $E_{\mathrm{T}}[\mathrm{N}]$ of free $\mathrm{Ga}$ and $\mathrm{N}$ atoms, respectively, and the optimized total energy, $E_{\mathrm{T}}[\mathrm{GaN}]$ per $\mathrm{Ga}-\mathrm{N}$ pair of a specific allotrope. The higher the positive $E_{\mathrm{c}}$, the stronger is the binding. By subtracting the cohesive energy of the structure in the global minimum (namely cohesive energy of 3D wurtzite structure) from that calculated for other $3 \mathrm{D}$ and $2 \mathrm{D}$ allotropes, one obtains the formation energy $E_{\mathrm{f}}$ at $T=0 \mathrm{~K}$ relative to the global minimum. A specific allotrope can correspond to a local minimum on the Born-Oppenheimer surface and hence can remain stable in spite of the fact that it has a negative $E_{\mathrm{f}}$. The average binding energy of layers in a multilayer structure can be expressed as $E_{\mathrm{i}}=E_{\mathrm{T}}[\mathrm{SL} \mathrm{GaN}]-E_{\mathrm{T}}[\mathrm{ML} \mathrm{GaN}]$ relative to constituent SL GaN. Cohesive, formation and binding energies are given per $\mathrm{Ga}-\mathrm{N}$ pair. The characters of bonds are analyzed by the contour plots of the total charge density, $\rho_{\mathrm{T}}$, the difference charge density (i.e. $\Delta \rho=\rho_{\mathrm{T}}-\rho_{\mathrm{Ga}, \mathrm{N}}$ ). The interionic charge transfer between $\mathrm{Ga}$ and $\mathrm{N}$ in different allotropes and the bond orders (BO) are calculated based on the DDEC6 method. ${ }^{31,32}$

The dynamical stability of the $2 \mathrm{D}$ and periodic allotropes is tested by $a b$ initio phonon calculations. ${ }^{33}$ A structure having positive vibrational frequencies, $\Omega(k)>0$, for all $k$ in the Brillouin zone, assures dynamical stability at $T=0 \mathrm{~K}$. Finite displacement method is employed using the Phonopy code, ${ }^{34}$ with $4 \times 4 \times 1$ and $2 \times 2 \times 2$ rectangular cell samplings for the bilayer and $3 \mathrm{D}$ periodic structures, respectively. The stability of the phases under thermal excitations is crucial, since an allotrope that is dynamically stable at $T=0 \mathrm{~K}$ can experience instability even at low temperatures. The stability of the $2 \mathrm{D}$ allotropes of GaN against thermal excitations is tested by performing finite-temperature ab initio molecular dynamics (MD) calculations. Accordingly, in the microcanonical ensemble, the velocities are scaled at each time step (of $2 \mathrm{fs}$ ) in order to keep the temperature constant, where we begin with $150 \mathrm{~K}$, and increase to 300 and $450 \mathrm{~K}$, each simulation time for each temperature lasting $2 \mathrm{ps}$, and $6 \mathrm{ps}$ in total. In specific cases, the temperature is raised up to $1000 \mathrm{~K}$. Moreover, MD simulations are repeated by using the canonical ensemble with Nosé thermostat, ${ }^{35}$ carried out at $150 \mathrm{~K}, 300 \mathrm{~K}$, and $450 \mathrm{~K}$.

In addition to the standard-DFT results, the calculations undertaking hybrid functionals (HSE, where a quarter of the exact exchange is incorporated in the XC functional) ${ }^{36-38}$ are also included, in order to obtain the corrected band structures of 3D bulk and 2D layered GaN structures.

\section{Results and discussion}

Single-layer, free-standing, h-GaN is planar and constitutes the basic element of various structures treated in this study. For the sake of comparison, we reproduced its structural parameters, cohesive and formation energies at $T=0 \mathrm{~K}$, as well as the fundamental band gaps. Its $2 \mathrm{D}$ rectangular lattice parameters are $a=6.42$ and $b=5.57 \AA$, the cohesive energy is $E_{\mathrm{c}}=$ $8.29 \mathrm{eV}$, and the formation energy is $E_{\mathrm{f}}=-1.16 \mathrm{eV}$, with an 
indirect fundamental band gap of $E_{\mathrm{g}}=2.16 \mathrm{eV}$ calculated by $\mathrm{PBE},{ }^{26,27}$ which is raised to $3.42 \mathrm{eV}$ after the HSE correction. ${ }^{36-38}$

Among various stacking sequences, two SL's of h-GaN with a uniform interlayer spacing of $\sim 2.47 \AA$ in $\mathrm{AA}^{\prime}$ conformation, which is specified as planar bilayer (B-PL), have a cohesive energy of $E_{\mathrm{c}}=8.57 \mathrm{eV}$ (Fig. 1). This interlayer spacing, which is larger than the usual $\mathrm{Ga}-\mathrm{N}$ bond distance $(1.88 \AA)$, does not allow the vertical bond formation, hence the layers are bound by weak vdW attraction. The binding energy of two layers relative to the free SL h-GaN is $280 \mathrm{meV}$ per Ga-N pair; which is not high. In order to eliminate any constraints due to the small cell size, the structural optimizations were performed in a cell including $8 \mathrm{Ga}$ and $8 \mathrm{~N}$ atoms. Furthermore, dynamical and thermal stability tests including vdW interactions indicate that B-PL is indeed a stable structure, despite its negative formation energy of $E_{\mathrm{f}}=-0.87 \mathrm{eV}$. Incidentally, at $T=450 \mathrm{~K}$ the planarity ceases; random and transient vertical bonds start to form between the two layers. The structure appears to be in a local minimum in the Born-Oppenheimer energy surface, since it did not transform spontaneously into a different one like the haeckelite structure, which was found to have lower energy. ${ }^{16}$ This relaxation was repeated in a $(4 \times 4)$ super cell to avoid any restrictions that may arise from the unit cell size, whereby no spontaneous transition took place.

In order to clarify this paradoxical situation, this time we started from the buckled h-GaN displaying a haeckelite-like structure (B-HK) from the side view, consisting of octagons and squares. The optimized structure has strong lateral bonds of $d_{1}=1.85-1.88 \AA$. However, weak vertical bonds of $d_{\mathrm{v}}=2.30 \AA$ formed between specific $\mathrm{Ga}$ and $\mathrm{N}$ atoms in adjacent layers, while the distance between other $\mathrm{Ga}$ and $\mathrm{N}$ atoms increased to $2.87 \AA$ (Fig. 1). Eventually, the B-HK structure was revealed as only $5 \mathrm{meV}$ energetically more favorable relative to B-PL. This structure is found to be stable also based on the dynamical stability analysis at $T=0 \mathrm{~K}$ and thermal stability analysis at $T=$ 450 K (Fig. S1, ESI†).

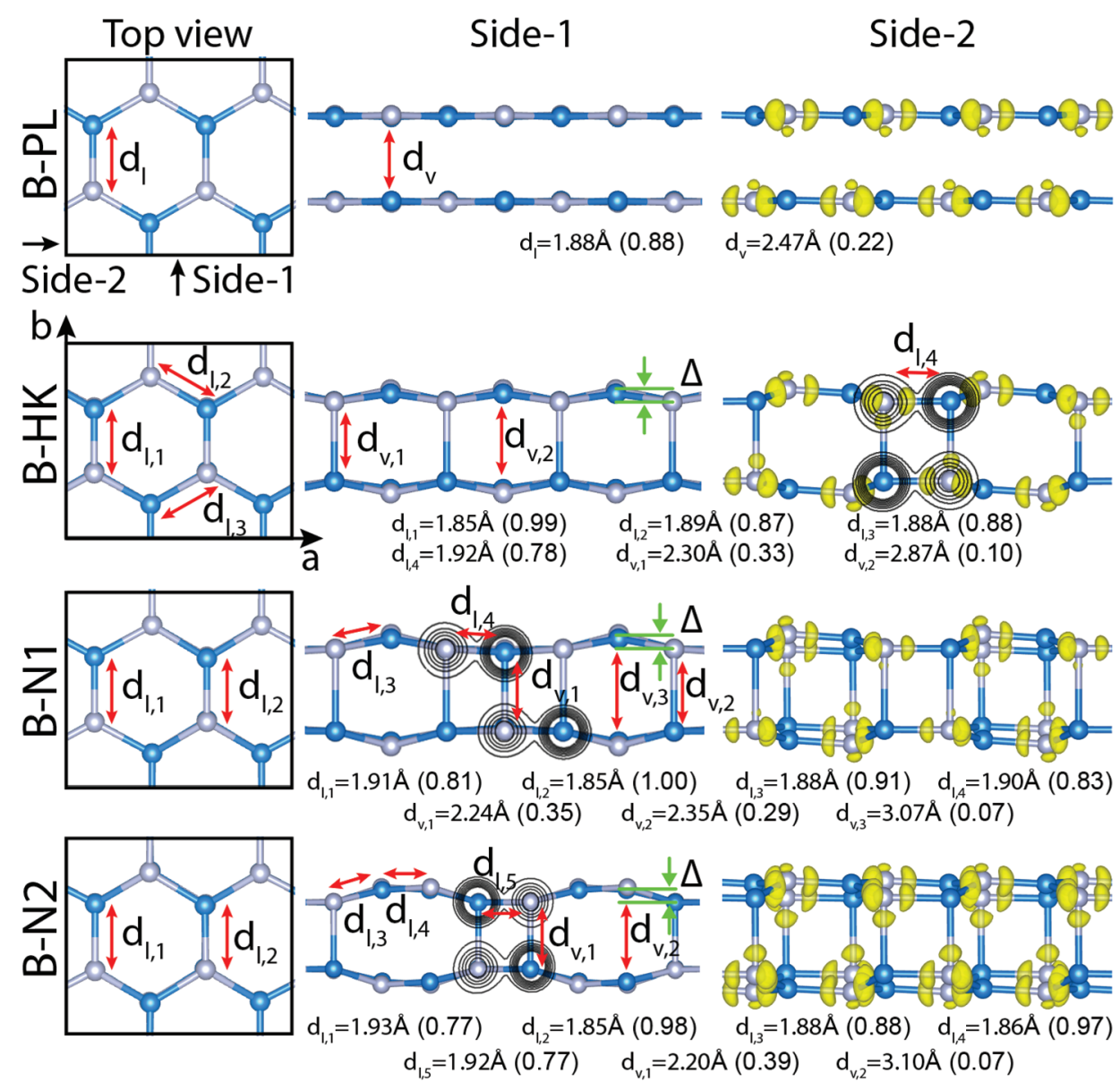

Fig. 1 Characterization of the allotropes of the GaN bilayer: planar, B-PL; haeckelite-like, B-HK; new allotropes, B-N1; B-N2. Atomic configurations: Left panels: Top view in the rectangular unit cell comprising $8 \mathrm{Ga}-\mathrm{N}$ pairs. Ga and $\mathrm{N}$ atoms are shown by gray and blue atoms. $a$ and $b$ are lattice constants. Middle panels: Side view-1 along $y$-direction. Contour plots of $\rho_{\mathrm{T}}$ show that higher charge density is shifted towards the $\mathrm{N}$ atom. Right panels: Side view-2 along $x$-direction. Isosurfaces of the difference charge density, $\Delta \rho$ indicates strong and weak bond charges of the lateral and vertical bonds, respectively. Ionic character of the bond becomes apparent with the bond charges shifting towards the anion. Optimized values of the lateral, $d_{1}$ and vertical, $d_{\mathrm{v}}$ bonds and their calculated bond orders (in parenthesis) are given for each bilayer structure. Isosurface values are set to $0.22 e$ bohr $^{-3}$. 
Our search for the allotropes of bilayers resulted in two new, stable, bilayer configurations, specified as B-N1 and B-N2 as shown in Fig. 1; both of them having cohesive energies even higher than that of B-HK. In particular, the B-N2 structure is $17 \mathrm{meV}$ (or $136 \mathrm{meV}$ per cell) energetically more favorable than B-HK. In Fig. 1, we present the atomic configurations, structural parameters, and charges of the lateral and vertical bonds of all four structures of GaN bilayers. The perspective views of the four bilayer structures are shown in Fig. S2, ESI. $\uparrow$ While all phases have similar top views of a hexagonal structure, side views differ significantly. The vertical bonds, which start to form between specific $\mathrm{Ga}$ and $\mathrm{N}$ atoms in different layers, are of crucial importance and are examined in terms of the isosurfaces of the difference charge, contour plots and bond orders $(\mathrm{BO})^{31,32}$ (the latter as a measure for the strength of a bond) in Fig. 1. In bilayers, the vertical bonds are longer than the usual in-plane lateral Ga-N bonds. Accordingly, the bond orders relevant to the lateral, in-plane Ga-N bonds are as high as 0.88 . The distance between $\mathrm{Ga}$ and $\mathrm{N}$ atoms in different planes of B-PL is $2.47 \AA$ and the corresponding BO is 0.22 .

In $\mathrm{B}-\mathrm{HK}$, the vertical bonds with $d_{\mathrm{v}}=2.30 \AA$ and $\mathrm{BO}=0.33$ are still longer and hence weaker than the lateral Ga-N bonds, nonetheless shorter than the interlayer distance of B-PL. The most energetic bilayer, B-N2 has the shortest vertical bond, $d_{\mathrm{v}}=$ $2.20 \AA$, at the same time the highest bond order, $\mathrm{BO}=0.39$ among four bilayer structures. Additionally, molecular dynamics (MD) simulations of the B-N2 structure sustained at $1000 \mathrm{~K}$, except that the positions of the vertical bonds change randomly in the course of the simulation. In the light of this discussion, one can argue that the vertical bonds set in bilayers are longer, and differ from the usual, strong Ga-N bonds. Generally, their length and strength determine the stability or energetics of the structure. Nevertheless, as the number of layers increases in multilayers, the vertical bonds are further shortened and eventually attain the strength of the bulk bonds. Incidentally, the cohesive energy of trilayer T-HK becomes higher than that of T-PL, as well as T-N2. In Table 1 the optimized values of structure parameters, bond orders, energetics and energy band gaps of bilayer and 3D periodic structures are presented.

Four bilayer structures undergo interesting structural transitions under biaxial strain/stress. This suggests that these structures are in local minima separated by small energy bar- riers. Fig. 2 summarizes the structural phase changes among the bilayer phases under biaxial strain. If one starts with the haeckelite-like structure B-HK, the variation of energy initially follows a parabolic curve, however drops suddenly at $a=6.53 \AA$ by $\sim 80 \mathrm{meV}$. At this point, the structure transforms to B-N1. As the strain continues to increase, the energy increases by following a steeper parabolic curve. When $a>6.56 \AA$, the energy raises suddenly by $\sim 70 \mathrm{meV}$ and the structure transforms to B-PL. Thereafter, the energy continues to increase. However, if one starts from the B-N2 structure with $a=6.46 \AA$, the energy increases without any phase transitions until $a=6.65 \AA$, where the phase changes to the planar B-PL following a small drop in energy as shown by the inset in Fig. 2 .

Similar structural transformations or phase changes occur under electrostatic charging. In order to show phase changes mediated by electrostatic charging, we examined only a few specific cases within plausible charging limits. ${ }^{39}$ For example, B-PL displays interesting phase changes. When charged by $q=$ $-0.5 e$ per unit cell $(0.03 e$ per atom $)$ or $q=-1.0 e$ per unit cell (0.06e per atom), it changes to the $\mathrm{B}-\mathrm{N} 2$ structure; nonetheless preserves its planar geometry when charged by $q=1.0 e$ per

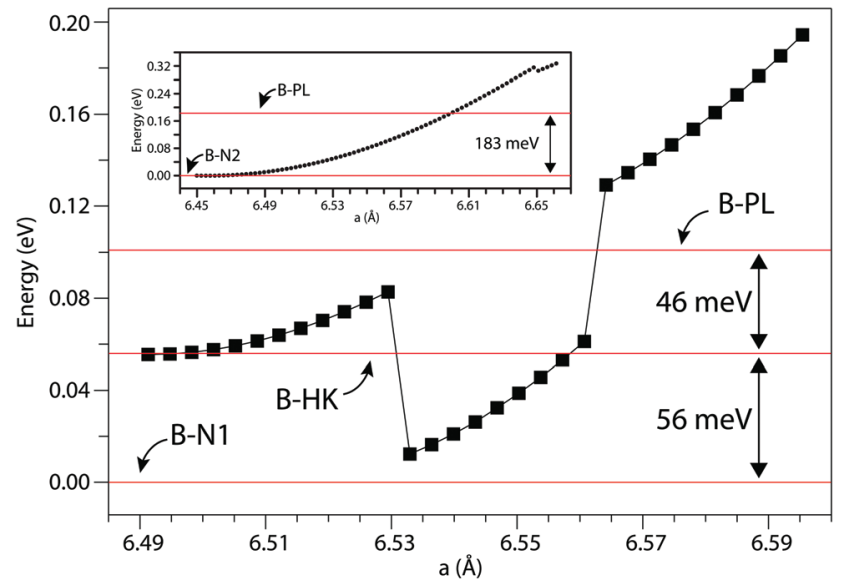

Fig. 2 Variation of the strain energy with lattice constants, leading to structural transformations of the bilayer structures. The lattice constants are obtained by applying uniform and tensile biaxial strain. Total energy (per cell) of the B-N1 structure is set to $0 \mathrm{eV}$. The transformation from $\mathrm{B}-\mathrm{N} 2$ to $\mathrm{B}-\mathrm{PL}$ is shown as an inset.

Table 1 Optimized values for monolayer (M), bilayer (B) and 3D periodic (P) structures of GaN: lattice parameters, $a, b$ and $c$ in $\AA$; cohesive energy $E_{\mathrm{c}}$ in eV per Ga-N pair; total interlayer interactions, $E_{\mathrm{i}}$ in eV per $\mathrm{Ga}-\mathrm{N}$ pair; formation energy $E_{\mathrm{f}}$ in eV per Ga-N pair; direct (d) or indirect (i) fundamental band gaps calculated by PBE and corrected by HSE, $E_{\mathrm{g}, \mathrm{d} / \mathrm{i}}$ in eV

\begin{tabular}{|c|c|c|c|c|c|c|c|c|c|}
\hline Allotrope & $a(\AA)$ & $b(\AA)$ & $c(\AA)$ & $\Delta(\AA)$ & $E_{\mathrm{c}}(\mathrm{eV})$ & $E_{\mathrm{i}}(\mathrm{eV})$ & $E_{\mathrm{f}}(\mathrm{eV})$ & $E_{\mathrm{g}, \mathrm{PBE}}-\mathrm{i}, \mathrm{d}(\mathrm{eV})$ & $E_{\mathrm{g}, \mathrm{HSE}}-\mathrm{i}, \mathrm{d}(\mathrm{eV})$ \\
\hline M-PL & 6.42 & 5.57 & - & - & 8.286 & 0.000 & -1.163 & $2.16(\mathrm{i})$ & $3.42(\mathrm{i})$ \\
\hline B-PL & 6.53 & 5.65 & - & - & 8.572 & 0.286 & -0.877 & $1.97(\mathrm{i})$ & $3.25(\mathrm{i})$ \\
\hline B-HK & 6.49 & 5.60 & - & 0.28 & 8.578 & 0.292 & -0.872 & $1.83(\mathrm{~d})$ & $3.11(\mathrm{~d})$ \\
\hline B-N1 & 6.51 & 5.63 & - & $0.06,0.41$ & 8.585 & 0.299 & -0.865 & 1.95(i) & $3.21(\mathrm{i})$ \\
\hline B-N2 & 6.46 & 5.61 & - & 0.45 & 8.595 & 0.309 & -0.854 & $1.96(\mathrm{~d})$ & $3.22(\mathrm{~d})$ \\
\hline P-PL & 6.62 & 5.74 & 4.88 & - & 8.941 & 0.655 & -0.508 & $1.23(\mathrm{~d})$ & $2.44(\mathrm{~d})$ \\
\hline P-HK & 6.43 & 5.50 & 5.50 & 0.75 & 9.325 & 1.039 & -0.125 & $1.82(\mathrm{~d})$ & $3.00(\mathrm{~d})$ \\
\hline $\mathrm{P}-\mathrm{N} 2$ & 6.42 & 5.63 & 5.76 & 0.86 & 9.063 & 0.777 & -0.387 & $1.79(\mathrm{~d})$ & $3.02(\mathrm{~d})$ \\
\hline WZ & 6.41 & 5.55 & 5.24 & 0.85 & 9.450 & 1.163 & 0.000 & $1.76(\mathrm{~d})$ & $3.03(\mathrm{~d})$ \\
\hline
\end{tabular}


unit cell and transforms to an intermediate state when charged by $q=0.5 e$ per unit cell. On the other hand, if one starts from the former negatively charged B-N2 phase, the structure remains in B-N2 when charged by $q=1.0 e$ per unit cell, and converts to an intermediate phase if it is charged by $q=2.0 e$ per unit cell ( $0.13 e$ per atom). B-HK does not transform into another phase when charged by $q= \pm 1.0 e$ per unit cell, however converts to an intermediate phase for $q=2.0 e$ per unit cell similar to the B-N2 case. B-HK, B-N1, and B-N2 preserve their geometries when charged by $q= \pm 0.5 e$ per unit cell. These preliminary tests suggest that the bilayer structures can be utilized as phase change materials and deserve further detailed analyses.

In Fig. 3, we present the energy bands of four bilayers calculated within PBE and corrected by HSE together with the iso-

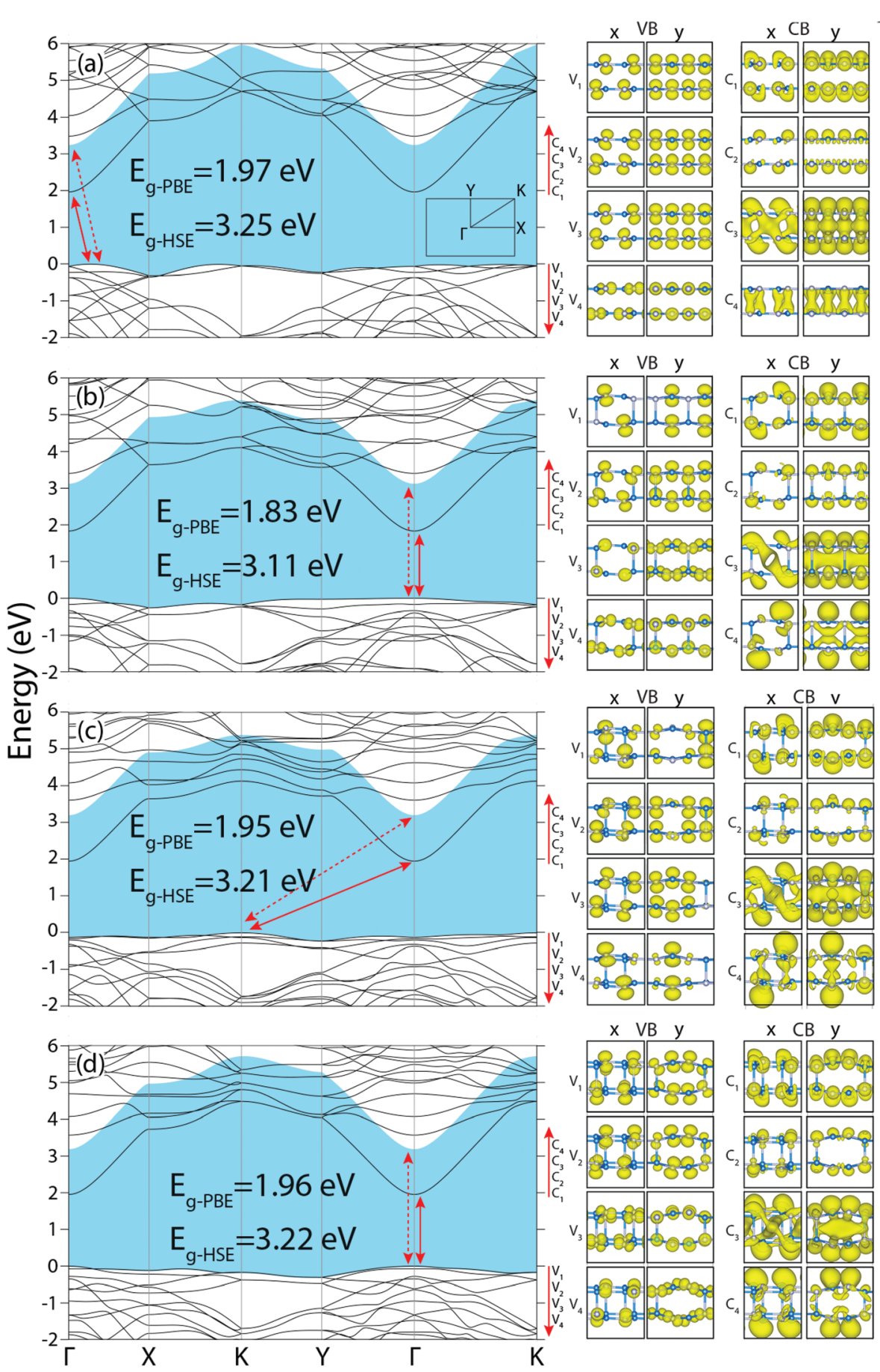

Fig. 3 Left: Energy band structures of four structures of bilayers: (a) B-PL, (b) B-HK, (c) B-N1 and (d) B-N2. The fundamental band gaps determined by the HSE correction over the PBE bands are shaded. Direct or indirect band gaps calculated by PBE and HSE are marked by arrows. Right: Isosurfaces of charge density of conduction, $C_{1}, C_{2} \ldots$ and valence band, $V_{1}, V_{2} \ldots$ states at the regarding extreme points in the Brillouin zone. 
surfaces of charge density of states at the band edges (the detailed charge density analysis and partial density of states are shown in Fig. S3 and S4, ESI $\dagger$ ). The four bilayer structures with honeycomb geometry in the top view share the rectangular Brillouin zone. It is surprising that, despite the differences in the bilayer structures, the electronic energy bands look similar. Fundamental band gaps of these four bilayer structures range between $3.11 \mathrm{eV}$ and $3.25 \mathrm{eV}$. Apparently, the HSE band gap of SL h-GaN being $3.42 \mathrm{eV}$ is reduced in bilayers due to the quantum size effect. The overall dispersions of the bands at the edge of the conduction band, in particular, near the center of the Brillouin zone appear to be similar. The bands at the edge of the valence band are generally flat, yet the slight maxima occur at different $k$-points. For example, in B-PL and B-N2 structures, the fundamental band gap is indirect due to a weak Mexican hat type dispersion around the $\Gamma$-point. Whereas, B-HK and B-N1 structures are direct band gap semiconductors. This direct-indirect band gap transition is an important feature among bilayer structures. The similarity of band dispersions at the band edges is revealed from the isosurfaces of charge density of the band states in Fig. 3. The bands at the edge of the valence band are constructed from $\mathrm{p}_{z}$-orbitals perpendicular to the bilayers having only weak lateral coupling; where coupling in the perpendicular direction is absent. This situation is seen as the main reason for their flat dispersion. On the other hand, the high dispersion of the conduction band edge is attributed to strong lateral coupling of the directional orbitals.

$3 \mathrm{D} \mathrm{GaN}$ in wurtzite structure is the allotrope of $\mathrm{GaN}$ in the global minimum among all allotropes in diverse dimensionality; each atom is fourfold coordinated where $\mathrm{Ga}-\mathrm{N}$ pairs form tetrahedrally coordinated mixed bonds. ${ }^{40}$ Another 3D allotrope is the zincblende structure, which has slightly higher energy. ${ }^{9}$ Here, with the premiss of revealing artificial structures with new functionalities, we explore the possibility of constructing $3 \mathrm{D}$ layered or quasi-layered structures as an alternative to the existing 3D crystals. Even if these layered structures were energetically less favorable, they can be stable in local minima and are expected to display properties different from those of $3 \mathrm{D}$ WZ. We considered 3D periodic new allotropes consisting of periodic planar (P-PL), periodic haeckelite (P-HK), and periodic N2 (P-N2). In Fig. 4 we show the optimized atomic configurations and bonding of these structures in the rectangular cell (perspective views of these structures are given as Fig. S5, ESI $\dagger$ ). $\mathrm{P}-\mathrm{PL}$ is a real layered structure with wide interlayer spacing. It is
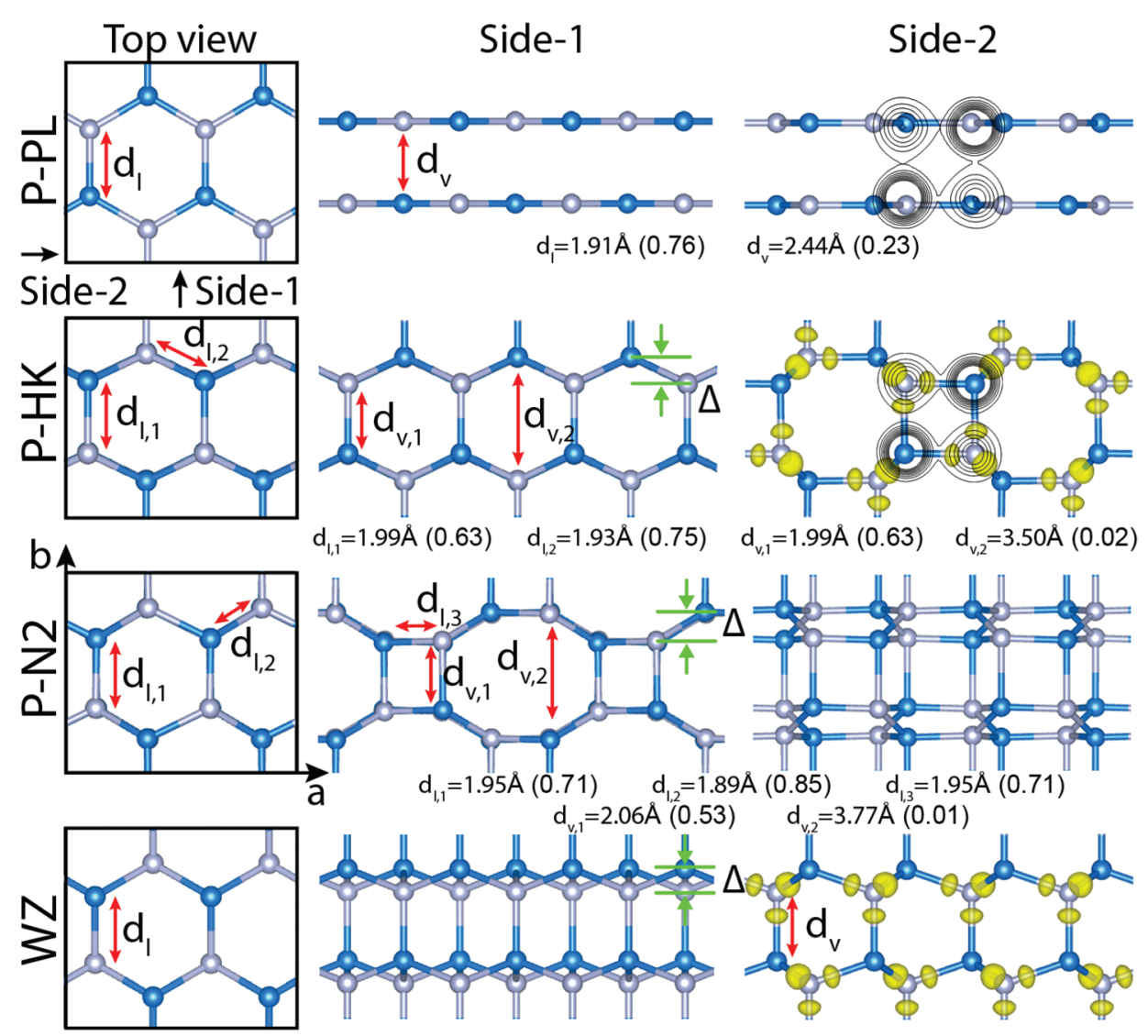

$d_{v, 1}=1.99 \AA(0.63)$

$\mathrm{d}_{\mathrm{v}, 2}=3.50 \AA(0.02)$

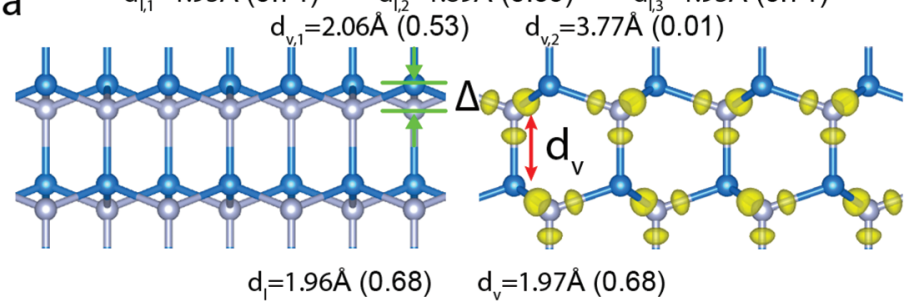

Fig. 4 Atomic structures and bond characterization of 3D (layered) periodic planar P-PL, haeckelite P-HK, P-N2, and wurtzite WZ structures. Left panels: Top view in the rectangular unit cell comprising $8 \mathrm{Ga}-\mathrm{N}$ pairs. $a$ and $b$ are lattice constants. Middle panels: Side view-1. Right panels: Side view-2. Isosurfaces of the difference charge density, $\Delta \rho$ are also shown. Optimized values of lateral $\left(d_{1}\right)$ and vertical $\left(d_{\mathrm{v}}\right)$ bonds and their calculated bond orders BO (in parentheses) are given for each structure. Isosurface values are set to $0.02 e$ bohr $^{-3}$. 
a vdW solid constructed from planar h-GaN layers in AA'A'A.. conformity and preserves three fold coordination with $\mathrm{sp}^{2}$-type hybridization. It is dynamically stable in $T=0 \mathrm{~K}$ (Fig. S6, ESI $\dagger$ ). However, it transforms to $3 \mathrm{D} \mathrm{WZ}$ structure at $T=150 \mathrm{~K}$. The P-HK structure is also dynamically stable at $T=0 \mathrm{~K}$ and remains stable even at $T=450 \mathrm{~K}$. In the side view, it displays a periodic arrangement consisting of octagons and squares. Its vertical bonds are contracted and attain the length close to that of the lateral bond, namely $d_{\mathrm{v} 1}=1.99 \AA$ with a high bond order of
$\mathrm{BO}=0.63$. All atoms are fourfold coordinated. Accordingly, it is considered a uniform crystal, except the holes or interstitial site of octagons. The cohesive energy of P-HK is $9.32 \mathrm{eV}$, which is $125 \mathrm{meV}$ energetically less favorable than WZ. P-N2 is $387 \mathrm{meV}$ energetically less favorable than WZ; it has vertical bonds of $d_{\mathrm{v}}=2.06 \AA$ and $\mathrm{BO}=0.53$. This structure is also dynamically stable, and remains stable even at $T=450 \mathrm{~K}$ (Fig. S6, ESI $\dagger$ ).

The energy band structures of the four 3D allotropes of GaN are presented in Fig. 5 (the detailed charge density ana-
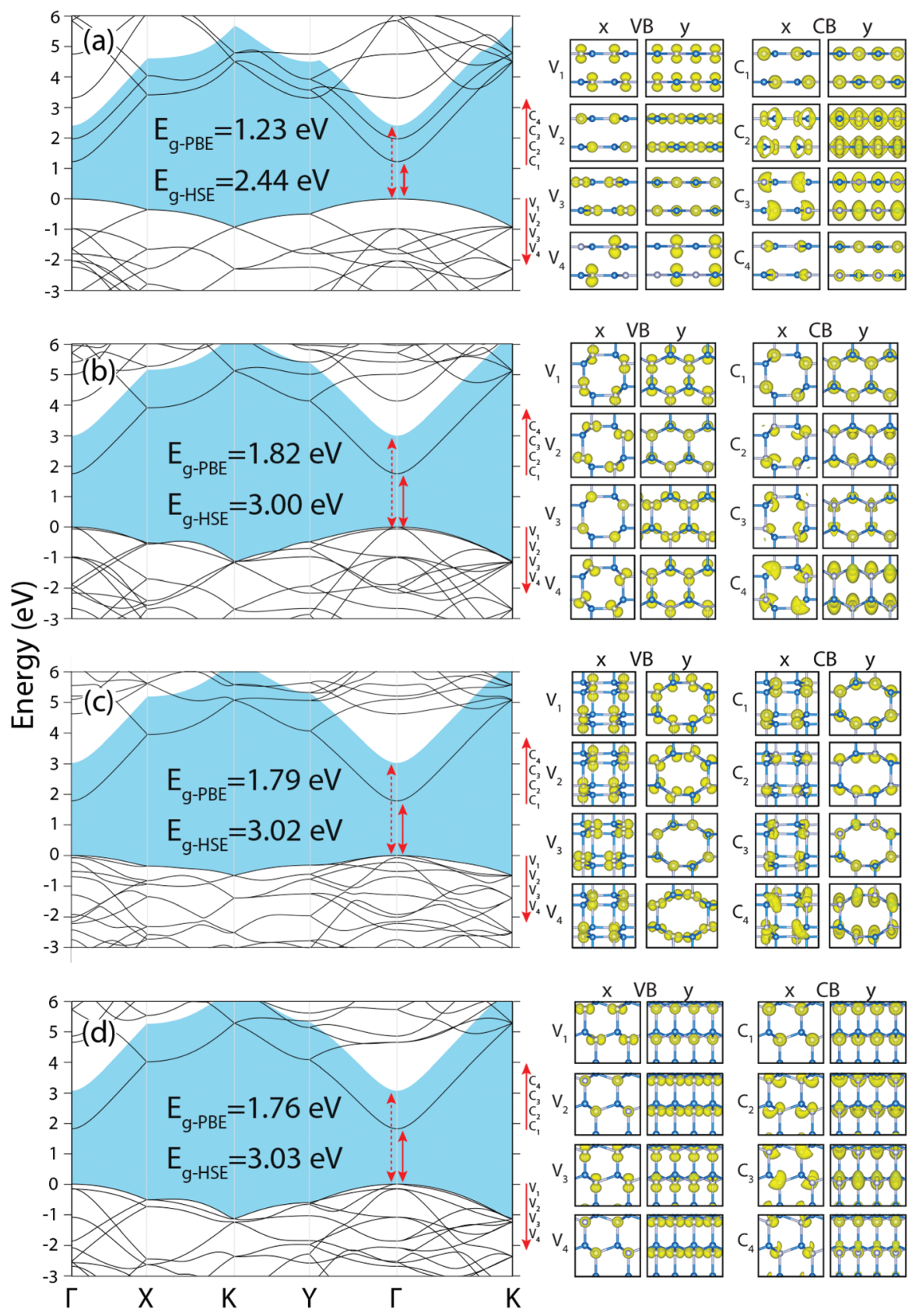

Fig. 5 Left: Energy band structures of four 3D structures of GaN. (a) Layered P-PL, (b) periodic haeckelite, P-HK, (c) P-N2 (new structure-2), (d) 3D WZ structure. Bands are calculated by PBE, and corrected by HSE. The fundamental band gaps determined by the HSE correction over the PBE bands are shaded. Direct or indirect band gaps are marked by arrows. Right: Isosurfaces of the charge density distributions of conduction, $\mathrm{C}_{1}, \mathrm{C}_{2}$, and valence band, $V_{1}, V_{2} \ldots$ states at the regarding extreme points in the Brillouin zone. 
lysis and partial density of states are shown in Fig. S7 and S8, ESI $\dagger$ ). Bands are calculated by PBE, subsequently corrected by HSE. All band structures look similar in the rectangular Brillouin zone. All 3D phases have direct fundamental band gaps. The band gap of the P-PL structure is $2.44 \mathrm{eV}$. The fundamental band gaps of the P-HK, P-N2 and P-WZ structures are, respectively, $3.00 \mathrm{eV}, 3.02 \mathrm{eV}$ and $3.03 \mathrm{eV}$; they are almost identical. The details of the band edge states are presented by their charge density isosurfaces in right panels of Fig. 5. The bands at the top of the valence band gained dispersion due to the periodicity perpendicular to the layers, nevertheless their dispersions are still lower than the dispersions of the bands at the bottom of the conduction band.

In summary, the previous discussion clarified an important question of whether stable layered vdW solids like graphite can be constructed by stacking of the planar, monolayer GaN, or if it will transform into different crystals by the formation of vertical bonds between $\mathrm{Ga}$ and $\mathrm{N}$ ions in adjacent layers, like silicite. By analyzing the character of the lateral and vertical bonds, we found that GaN is in between: among four different stable phases corresponding to the local minima in bilayer geometry (two of them newly predicted in this study), the energy difference between the planar bilayer bound by vdW attraction and the other three stable bilayers with weak vertical bonds is minute. Nevertheless, planar layers are buckled, even if the vertical bonds are longer and weaker than the usual GaN bonds. Among these four phases of bilayer GaN, the transitions are not spontaneous, but require external excitation like strain or electrostatic charging. Transitions between two specific phases bring about also the direct-indirect transition of the fundamental band gap.

With increasing layer numbers, vertical bonds become shorter and stronger; eventually approaching the lateral GaN bonds in $3 \mathrm{D}$ crystals. In 3D, we found one layered vdW solid, predicted one new crystal having vertical bonds in addition to 3D wurtzite, zincblende and haeckelite structures. 3D layered (planar) GaN is dynamically stable, nevertheless transformed to wurtzite structure at $T=150 \mathrm{~K}$. Both the new crystal (P-N2) and the crystal in the haeckelite structure (P-HK) are dynamically stable and sustained thermal excitations at $450 \mathrm{~K}$. Both have wide direct band gaps of $\sim 3.0 \mathrm{eV}$, whereas are energetically less favorable than the wurtzite structure. Accordingly, h-GaN appears to be different from vdW solids like h-BN and homogeneous crystal of silicite; since in its multilayer forms, it can lead to both vdW solid and homogeneous crystals.

\section{Conflicts of interest}

There are no conflicts to declare.

\section{Acknowledgements}

The calculations were performed at TUBITAK ULAKBIM, High Performance and Grid Computing Center (TR-Grid
e-Infrastructure) and the National Center for High Performance Computing of Turkey (UHeM) under grant no. 5003622015. This work was supported by the Scientific and Technological Research Council of Turkey (TUBITAK) under Project No. 117F241. E. D. acknowledges support from the Turkish Academy of Sciences - Outstanding Young Scientists Award Program (TUBA-GEBIP). S. C. acknowledges financial support from the Academy of Sciences of Turkey (TUBA).

\section{References}

1 S. Nakamura, M. Senoh, S.-i. Nagahama, N. Iwasa, T. Matsushita and T. Mukai, InGaN-based laser diodes with an emission wavelength of $450 \mathrm{~nm}$, Appl. Phys. Lett., 2000, 76, 22-24.

2 S. Nakamura, Nobel Lecture: Background story of the invention of efficient blue InGaN, Rev. Mod. Phys., 2015, 87, 1139.

3 H. Morkoç, Handbook of nitride semiconductors and devices, Materials Properties, Physics and Growth, John Wiley \& Sons, 2009, vol. 1.

4 E. Durgun, S. Tongay and S. Ciraci, Silicon and III-V compound nanotubes: Structural and electronic properties, Phys. Rev. B: Condens. Matter Mater. Phys., 2005, 72, 075420.

5 H. Şahin, S. Cahangirov, M. Topsakal, E. Bekaroglu, E. Akturk, R. T. Senger and S. Ciraci, Monolayer honeycomb structures of group-IV elements and III-V binary compounds: First-principles calculations, Phys. Rev. B: Condens. Matter Mater. Phys., 2009, 80, 155453.

6 A. Onen, D. Kecik, E. Durgun and S. Ciraci, GaN: From three- to two-dimensional single-layer crystal and its multilayer van der Waals solids, Phys. Rev. B, 2016, 93, 085431.

7 S. Cahangirov, M. Topsakal, E. Aktürk, H. Șahin and S. Ciraci, Two- and one-dimensional honeycomb structures of silicon and germanium, Phys. Rev. Lett., 2009, 102, 236804 .

8 S. Cahangirov, M. Topsakal and S. Ciraci, Armchair nanoribbons of silicon and germanium honeycomb structures, Phys. Rev. B: Condens. Matter Mater. Phys., 2010, 81, 195120.

9 D. Kecik, A. Onen, M. Konuk, E. Gürbüz, F. Ersan, S. Cahangirov, E. Aktürk, E. Durgun and S. Ciraci, Fundamentals, progress, and future directions of nitridebased semiconductors and their composites in two-dimensional limit: A first-principles perspective to recent synthesis, Appl. Phys. Rev., 2018, 5, 011105.

10 D. Xu, H. He, R. Pandey and S. P. Karna, Stacking and electric field effects in atomically thin layers of GaN, J. Phys.: Condens. Matter, 2013, 25, 345302.

11 A. K. Singh and R. G. Hennig, Computational synthesis of single-layer GaN on refractory materials, Appl. Phys. Lett., 2014, 105, 051604.

12 N. Sanders, D. Bayerl, G. Shi, K. A. Mengle and E. Kioupakis, Electronic and Optical Properties of TwoDimensional GaN from First-Principles, Nano Lett., 2017, 17, 7345-7349. 
13 Z. Y. Al Balushi, K. Wang, R. K. Ghosh, R. A. Vilá, S. M. Eichfeld, J. D. Caldwell, X. Qin, Y.-C. Lin, P. A. DeSario, G. Stone, et al., Two-dimensional gallium nitride realized via graphene encapsulation, Nat. Mater., 2016, 15, 1166.

14 S. Cahangirov, V. O. Özçelik, A. Rubio and S. Ciraci, Silicite: The layered allotrope of silicon, Phys. Rev. B: Condens. Matter Mater. Phys., 2014, 90, 085426.

15 S. Cahangirov, V. O. Özçelik, L. Xian, J. Avila, S. Cho, M. C. Asensio, S. Ciraci and A. Rubio, Atomic structure of the $3 \times 3$ phase of silicene on Ag (111), Phys. Rev. B: Condens. Matter Mater. Phys., 2014, 90, 035448.

16 A. Kolobov, P. Fons, J. Tominaga, B. Hyot and B. Andre, Instability and spontaneous reconstruction of few-monolayer thick GaN graphitic structures, Nano Lett., 2016, 16, 4849-4856.

17 D. C. Camacho-Mojica and F. López-Urías, GaN haeckelite single-layered nanostructures: monolayer and nanotubes, Sci. Rep., 2015, 5, 17902.

18 H. Zhang, F.-S. Meng and Y.-B. Wu, Two single-layer porous gallium nitride nanosheets: A first-principles study, Solid State Commun., 2017, 250, 18-22.

19 P. A. Brown and K. L. Shuford, Archimedean (4, 8)-tessellation of haeckelite ultrathin nanosheets composed of boron and aluminum-group V binary materials, Nanoscale, 2016, 8, 19287-19301.

20 A. Kolobov, P. Fons, Y. Saito, J. Tominaga, B. Hyot and B. André, Strain engineering of atomic and electronic structures of few-monolayer-thick GaN, Phys. Rev. Mater., 2017, 1, 024003.

21 E. Gürbüz, S. Cahangirov, E. Durgun and S. Ciraci, Single layers and multilayers of GaN and AlN in square-octagon structure: Stability, electronic properties, and functionalization, Phys. Rev. B, 2017, 96, 205427.

22 P. E. Blöchl, Projector augmented-wave method, Phys. Rev. B: Condens. Matter, 1994, 50, 17953-17979.

23 G. Kresse and D. Joubert, From ultrasoft pseudopotentials to the projector augmented-wave method, Phys. Rev. B: Condens. Matter, 1999, 59, 1758-1775.

24 G. Kresse and J. Hafner, Ab initio molecular-dynamics simulation of the liquid-metal-amorphous-semiconductor transition in germanium, Phys. Rev. B: Condens. Matter, 1994, 49, 14251.

25 G. Kresse and J. Furthmüller, Efficient iterative schemes for ab initio total-energy calculations using a plane-wave basis set, Phys. Rev. B: Condens. Matter, 1996, 54, 11169-11186.
26 J. P. Perdew, K. Burke and M. Ernzerhof, Generalized gradient approximation made simple, Phys. Rev. Lett., 1996, 77, 3865.

27 K. Burke, J. Perdew and M. Ernzerhof, Generalized Gradient Approximation Made Simple [Phys. Rev. Lett. 77, 3865 (1996)], Phys. Rev. Lett., 1997, 78, 1396.

28 S. Grimme, Semiempirical GGA-type density functional constructed with a long-range dispersion correction, J. Comput. Chem., 2006, 27, 1787-1799.

29 T. Bucko, J. Hafner, S. Lebegue and J. G. Angyán, Improved description of the structure of molecular and layered crystals: ab initio DFT calculations with van der Waals corrections, J. Phys. Chem. A, 2010, 114, 11814-11824.

30 H. J. Monkhorst and J. D. Pack, Special points for Brillouin-zone integrations, Phys. Rev. B: Solid State, 1976, 13, 5188.

31 N. G. Limas and T. A. Manz, Introducing DDEC6 atomic population analysis: part 2 . Computed results for a wide range of periodic and nonperiodic materials, RSC Adv., 2016, 6, 45727-45747.

32 T. A. Manz, Introducing DDEC6 atomic population analysis: part 3. Comprehensive method to compute bond orders, RSC Adv. , 2017, 7, 45552-45581.

33 S. Baroni, S. De Gironcoli, A. Dal Corso and P. Giannozzi, Phonons and related crystal properties from density-functional perturbation theory, Rev. Mod. Phys., 2001, 73, 515.

34 A. Togo and I. Tanaka, First principles phonon calculations in materials science, Scr. Mater., 2015, 108, 1-5.

35 S. Nosé, A unified formulation of the constant temperature molecular dynamics methods, J. Chem. Phys., 1984, 81, 511-519.

36 J. Heyd and G. E. Scuseria, Efficient hybrid density functional calculations in solids: Assessment of the HeydScuseria-Ernzerhof screened Coulomb hybrid functional, J. Chem. Phys., 2004, 121, 1187-1192.

37 J. Heyd and G. E. Scuseria, Assessment and validation of a screened Coulomb hybrid density functional, J. Chem. Phys., 2004, 120, 7274-7280.

38 J. Paier, M. Marsman, K. Hummer, G. Kresse, I. C. Gerber and J. G. Ángyán, Screened hybrid density functionals applied to solids, J. Chem. Phys., 2006, 124, 154709.

39 A. Pachoud, M. Jaiswal, P. K. Ang, K. P. Loh and B. Ozyilmaz, Graphene transport at high carrier densities using a polymer electrolyte gate, $E P L, 2010,92,27001$.

40 W. A. Harrison and S. Ciraci, Bond-orbital model. II, Phys. Rev. B: Solid State, 1974, 10, 1516. 